Relations industrielles

Industrial Relations

\title{
Le droit du travail
}

\section{Marie-Louis Beaulieu}

Volume 14, numéro 3, juillet 1959

URI : https://id.erudit.org/iderudit/1022291ar

DOI : https://doi.org/10.7202/1022291ar

Aller au sommaire du numéro

\section{Éditeur(s)}

Département des relations industrielles de l'Université Laval

ISSN

0034-379X (imprimé)

1703-8138 (numérique)

Découvrir la revue

Citer ce document

Beaulieu, M.-L. (1959). Le droit du travail. Relations industrielles / Industrial Relations, 14(3), 414-418. https://doi.org/10.7202/1022291ar

Tous droits réservés @ Département des relations industrielles de l’Université Laval, 1959
Ce document est protégé par la loi sur le droit d'auteur. L’utilisation des services d'Érudit (y compris la reproduction) est assujettie à sa politique d'utilisation que vous pouvez consulter en ligne.

https://apropos.erudit.org/fr/usagers/politique-dutilisation/ 


\title{
Le droit du travail
}

\section{Marie-Louis Beaulieu}

Bien que notre Droit du Travail, après s'être d'abord inspiré du Droit français, soit de plus en plus canadien d'origine, le Traité publié l'an dernier par le professeur A. Brun, de la Faculté de Droit de Lyon, et $\mathrm{Me} \mathrm{H}$. Galland, Avocat au Conseil d'Etat et à la Cour de Cassation, présente pour nous beaucoup d'intérêt. ${ }^{1}$ J'entends par là qu'il peut rendre de grands services à nos étudiants et aussi à la magistrature et aux membres du Barreau.

Dans la préface de ce bel ouvrage, pour conclure la synthèse qu'il fait des «deux préoccupations essentielles, éventuellement contradictoires, dominant l'âge industriel que nous vivons: un souci croissant du rendement de la production, mais en même temps une défense de mieux en mieux organisée des droits individuels et collectifs du travailleur, "André Siegfried écrit: ${ }^{2}$

\begin{abstract}
En étudiant le Droit du Travail, M. Brun et M. Galland, experts en la matière, ont surivi pas à pas, et dans tous ses détails, la répercussion juridique de l'étonnante révolution technique, économique et sociale qu'a subie depuis deux siècles le monde contemporain. Je ne crois pas qu'on eût pu choisir sujet plus passionnant, ni dont la portée soit plus considérable. Je n'en connais pas qui prête à plus de réflexions sur la nature et l'évolution de notre structure sociale, sur les tendances profondes d'une destinée, dont Valéry disait qu'elle constiiuait \& une grande aventure $»$. Voilà donc un travail utile et j'ajoute fait de main d'ouvrier.
\end{abstract}

C'est un très beau témoignage de la part du grand académicien qui vient de mourir et que Roger Seyldoux qualifiait récemment de "Maître à penser d'une génération ».

Dans l'avant-propos, les auteurs ont cru « utile d'attirer l'attention du lecteur sur quelques-unes des idées essentielles qui inspirent la conception de l'ouvrage et son plan $»$. Trois de ces idées doivent faire l'objet de notre méditation. Nous aurions voulu les commenter et en faire l'application chez nous autrement que par quelques phrases mais cela dépasserait l'espace alloué à notre rubrique «LIVRES ET REVUES ».

La première idée essentielle, et c'est la plus importante car elle fait beaucoup plus que constater un état de chose que les auteurs vont exposer et analyser, elle nous indique la portée de l'ouvrage et toute une philosophie du Droit du Travail. ${ }^{3}$ «Les rapports collectifs de

(1) Brun, A., de la Faculté de Droit de Lyon, et Galland, H., avocat au Conseil d'Etat et à la Cour de Cassation. Droit du travail, Sirey, 1958, Paris, 1053 pp.

(2) p. 4.

(3) pp. 5 et 6. 
travail et la tutelle de l'état ont pris une importance toujours grandissante. Il en résulte apparemment un certain déclin des rapports individuels de travail et du rôle joué par le contrat qualifié par les juristes classiques de «louage de services». Ainsi des auteurs ont-ils pu estimer que le contrat individuel de travail classique était une forme juridique périmée. Cependant, nous ne partageons pas ce point de vue. Le présent ouvrage a pour objet d'exposer le « Droit positif français », c'est-à-dire le Droit appliqué; or, à cet égard, qu'on le veuille ou non, les constatations suivantes s'imposent:

"1-Le contrat de travail reste le cadre fondamental dans lequel s'intègrent les dispositions légales et règlementaires. C'est la technique juridique traditionnelle qui demeure prépondérante».

«2-La Cour de Cassation, régulatrice de notre jurisprudence et interprète souveraine du Droit, reste très fermement attachée à la notion de contrat individuel de travail; elle caractérise même ce contrat par le vieux critère du «lien de subordination».

«3-Les accords sociaux ne suppriment pas les contrats individuels et les mesures qu'ils renferment s'insèrent dans ces contrats si bien que leur application relèvera souvent de la technique habituelle des obligations ».

«4-Sur un plan moins général, il ne faut pas sous-estimer un aspect particulier de l'évolution sociale contemporaine. $\mathrm{Si}$ on limite son contenu aux obligations résultant de l'accond des volontés, le contrat individuel de travail est certes en régression, lorsqu'il s'agit des salariés ordinaires, mais il a toujours un rôle important quant aux salariés supérieurs. Dans un monde industriel qui est à la veille d'une nouvelle révolution - l'automation élimine peu à peu les besognes matérielles - il est permis de croire que le contrat individuel de travail n'a pas dit son dernier mot. Il contribue et contribuera à la défense de la personnalité contre les forces qui la menacent».

Les auteurs concluent: "Pour ces diverses raisons nous avons laissé une large place au contrat de travail - tel qu'il est aménagé par l'action simultanée des lois, des conventions collectives, des coutumes professionnelles, de l'entente des parties».

Le point de vue du Professeur Brun et de Me Galland est celui que nous trouvons dans la jurisprudence de nos tribunaux judiciaires et c'est aussi celui du Barreau, sauf de la jeune génération. La jurisprudence arbitrale et l'enseignement de nos professeurs sont aussi de tendance opposée. Sans oublier que le contrat de travail reste le cadre fondamental dans lequel s'intègrent les dispositions légales et règlementaires, et sans professer que les accords sociaux suppriment les contrats individuels, pour notre part, nous croyons que les rapports 
collectifs oocupent une plus grande place que celle que l'ouvrage leur fait.

Où je partage entièrement le point de vue des auteurs, c'est d'avoir voulu exposer «le droit appliqué ». Dans la préface de la première édition de ce qu'il a appelé «COURS DE DROIT CIVIL POSITIF FRANÇAIS », Josserand a écrit, il y a trente ans:

Le droit que nous avons pris pour objet de notre étude est avant-tout le droit jurisprudentiel, c'est-à-dire celui qui se réalise: nous avons entendu faire de la science et non du roman.

Deux ans plus tard, à l'avant-propos de la deuxième édition, l'ancien Doyen de la Faculté de Droit de Lyon écrivait, en parlant de l'accueil réservé à son Oeuvre:

Il semble donc bien que la formule dont-elle s'inspire réponde à une préoccupation universelle: c'est le droit vivant que l'on veut connaître, non un droit mort.

Le Professeur Brun est de Lyon, comme l'on sait. Peut-être a-t-iI été l'élève de Josserand. A tout événement, l'ouvrage qu'il a écrit avec Me Galland, bien que de facture tout à fait différente de celle du grand civiliste lyonnais, est un droit pratique, un droit bien vivant.

La deuxième idée essentielle qui se dégage de l'ouvrage, c'est que «le droit français du Travail tend à devenir très diversifié d'une manière d'ailleurs excessive». Bien que beaucoup moins considérable, notre Droit du Travail est déjà lui aussi très diversifié. Enfin, les auteurs nous montrent tout au cours de leur traité comment le droit du travail français "participe activement au vaste mouvement universaliste qui tend à l'établissement des règles du travail communes à diverses nations ». Il en est de même du droit ouvrier québécois.

Voyons maintenant le plan de l'ouvrage. Nous le trouvons dans l'introduction, après l'objet, l'importance, la délimitation et l'aspect général du Droit du Travail: ${ }^{4}$

Première Partie: Les données fondamentales du Droit du Travail.

Deuxième Partie: Les rapports individuels de travail, le Contrat de

Travail.

Troisième Partie: Les rapports collectifs de travail.

C'est le plan logique de l'Ouvrage de cette nature et il est commandé par plusieurs idées, lisons-nous dans l'explication que les auteurs nous en donnent: ${ }^{5}$

Les rapports de travail se nouent sur deux plans différents. Tantôt les relations s'instaurent entre un patron et un salarié déterminés. Elles donnent naissance à un contrat individuel de travail qui, aujourd'hui, est modelé non plus seulement par la volonté des parties, mais enoore

(4) p. 13.

(5) p. 11 . 
et surtout par l'autorité publique. Si des difficultés surgissent à l'occasion de ce contrat elles constituent un conflit individuel de travail.

Tantôt les relations s'établissent sur une base collective. Les patrons comme les salariés, se groupent et forment des syndicats qui élaborent des ententes de grande envergure: les conventions collectives. Des difficultés éclatent-elles entre les forces en présence, le conflit prend un caractère collectif et il se traduira soit par des solutions de force, la grève ou le lock-out, soit par une procédure de règlement pacifique: médiation, arbitrage.

Mais, écrivent les auteurs,

Il apparait nécessaire, avant d'analyser les textes du Droit positif, de présenter le climat dans lequel le Droit du Travail évolue et ses principes généraux. On est ainsi conduit à adopter les trois parties suivantes.

Et les auteurs donnent le plan que nous venons de reproduire.

Dans la première partie divisée en quatre titres: l'évolution historique, les sources, les organismes administratifs et les juridictions, les caractéristiques du Droit du Travail, nous voulons surtout signaler à nos lecteurs les sources du Droit du Travail, en partioulier les sources étatiques, les sources professionnelles et les conflits entre les diverses sounces de ce droit, non pas que les principes constitutionnels, les lois, les règlements, le droit jurisprudentiel de même que les coutumes, les usages et les institutions syndicales que les auteurs exposent et commentent peuvent s'appliquer chez nous, mais parce que nous y trouvons une foule de renseignements d'une grande utilité dans l'élaboration de notre droit, et que le droit du travail québécois participe au vaste mouvement qui tend à l'établissement de règles communes à diverses nations, comme nous venons de l'écrire.

De tout l'ouvrage, c'est la deuxième partie qui nous intéresse davantage et peut surtout nous servir: les relations individuelles et le contrat du travail. Ici, nous avons du droit civil selon des conceptions nouvelles, droit civil adapté aux conditions sociales et aux exigences économiques du temps mais, avec comme droit commun les principes et les règles du code civil. Nous voulons signaler à nos lecteurs la structure du contrat de travail et ses caractères juridiques: l'autonomie du contrat individuel de travail, en particulier, la distinction entre le contrat de travail et le contrat d'entreprise; les règles de formation du contrat, son contenu, son exécution, enfin, l'extinction et la suspension du contrat de travail. Cette deuxième partie se termine par l'étude du contrat d'apprentissage, la formation et l'orientation professionnelles.

Nous savons déjà que la troisième partie est consacrée aux rapports collectifs de travail. C'est d'abord le idroit et le mouvement syndical, 
les conventions collectives. Il est vrai qu'après être allé, en 1924, chercher notre première inspiration en France, pour rédiger la section III de la Loi des Syndicats Professionnels, ${ }^{6}$ depuis 1934, d'abord avec la Loi relative à l'extension juridique des conventions collectives de travail $^{7}$ et davantage depuis 1944 avec la Loi des relations ouvrières, ${ }^{8}$ nos lois de conventions collectives sont soit d'origine canadienne soit d'inspiration américaine. Cependant, il est utile de connaître le Droit du Travail français en matière de syndicats professionnels et d'ententes collectives.

Un titre entier porte sur l'entreprise et les institutions collectives. Nos relations patronales-ouvrières diffèrent beaucoup de ce qui se pratique en France. Nous avons des usages et des techniques canadiennes, ou américaines, mais je conseille à nos chefs d'entreprise et à leurs conseillers et aviseurs de lire les pages que les auteurs écrivent sur le sujet. Ils y trouveront des formules propres à les inspirer, en particulier ce qui a trait aux relations entre le chef d'entreprise et le personnel, et les Comités d'entreprise qu'on a pratiqués chez nous avec profit durant la guerre.

Au tout début de sa préface, Siegfried écrit:

Tout phénomène humain comporte un aspect juridique sous l'angle duquel, à un moment domné, il convient nécessairement de l'envisager. C'est dans ces conditions que le droit du travail reflète directement la formidable transformation qu'a provoquée, dans nos sociétés occidentales, la révolution du machinisme. La portée d'un livre consacré à paneil sujet ne peut-être que considérable et je ne crois pas exagérer en disant qu'il s'agit ici d'une étude fondamentale.

Etude fondamentale en effet que le Droit du Travail du Professeur Brun et de Me Galland et nous le recommandons a tous ceux chez nous qui s'intéressent aux relations patronales-ouvrières.

(6) S.R.Q. (1941) ch. 162.

(7) 24, Geo. V, ch. 56.

(8) S.R.Q. (1941) ch. 162a. 